Rev. Adm. Saúde Vol. 17, № 68, Jul. - Set. 2017

http://dx.doi.org/10.23973/ras.68.50

ARTIGO ORIGINAL

\title{
Satisfação do paciente idoso com internação em um hospital universitário
}

Satisfaction of the elderly patient with care in a university hospital

Rilva Lopes de Sousa-Muñoz ${ }^{1}$, Marianne Silveira Mendonça ${ }^{2}$, Vanessa Cruz $^{2}$, Werton Sales ${ }^{2}$, Victor Egypto Pereira², Ângela Siqueira de Figueiredo ${ }^{1}$

1. Médica. Docente do Centro de Ciências Médicas da Universidade Federal da Paraíba

2. Estudante de graduação em medicina do Centro de Ciências Médicas da Universidade Federal da Paraíba

\section{RESUMO}

O objetivo deste estudo foi avaliar a satisfação de 102 pacientes idosos com sua internação em enfermarias de clínica médica de um hospital universitário através de estudo observacional mediante entrevistas diretas. Os pacientes ficaram satisfeitos com a assistência hospitalar, a equipe médica e de enfermagem, assim como com o resultado do tratamento e as condições de limpeza e ruído nas enfermarias, porém poucos relataram obtenção de informações sobre sua doença e tratamento pela equipe de saúde.

Palavras-chave: Idoso. Hospitalização. Satisfação do paciente.

\section{ABSTRACT}

This study aims to evaluate the satisfaction of 102 elderly patients with their hospitalization in medical infirmaries of a university hospital through observational study through direct interviews. Patients were satisfied with hospital care, medical and nursing staff, as well as the outcome of treatment, 
cleaning conditions and noise on the infirmaries, but few reported obtaining information about their disease and treatment by the health professionals.

Keywords: Elderly. Hospitalization. Patient satisfaction.

\section{INTRODUÇÃO}

Muitos profissionais e gestores de saúde em hospitais gerais não atribuem a devida importância à provisão inclusiva de serviços hospitalares para pessoas de idade avançada ${ }^{1}$. Contudo, o envelhecimento populacional brasileiro é uma realidade, por isso os gestores de serviços de saúde precisam estar atentos às necessidades e demandas impostas pelo contingente progressivamente maior de pacientes idosos na sua clientela.

As internações hospitalares da população idosa tiveram grande frequência na última década no Brasil, chegando a representar um terço do total de hospitalizações e apresentando prolongados períodos de permanência². Contudo, na maioria dos hospitais públicos brasileiros, a estruturação do atendimento hospitalar tem sido predominantemente voltada aos problemas da população adulta jovem, cujas características são diferentes daquelas apresentadas pelos idosos.

A satisfação do paciente é definida como a percepção de suas necessidades aliada ao atendimento de suas expectativas e à avaliação das características e dos resultados de sua experiência com o tratamento ${ }^{3}$, remetendo à realização da expectativa ou necessidade daquele para com o serviço utilizado ${ }^{4}$. A avaliação da satisfação com tratamentos médicos deve ser realizada também em relação a pacientes idosos, porém pouco se sabe sobre a opinião dos mais velhos sobre a assistência que lhes é prestada nos hospitais ${ }^{5}$. Esta perspectiva fornece informação essencial para aferir a qualidade dos serviços de um hospital, constituindo uma medida para sua melhoria ${ }^{6}$.

Com base nessas considerações, esta pesquisa teve como objetivo avaliar a satisfação de pacientes idosos com o atendimento recebido durante a internação em enfermarias de clínica médica de um hospital universitário, com enfoque na informação recebida pela equipe de saúde do serviço.

\section{MATERIAL E MÉTODOS}

O modelo deste estudo foi observacional e transversal, sendo realizado nas enfermarias de clínica médica do Hospital Universitário Lauro Wanderley (HULW), em João Pessoa, Paraíba, no período de setembro de 2014 a março de 2015. 
Incluíram-se 102 idosos internados consecutivamente nas enfermarias de clínica médica do HULW durante o período do estudo. Foram considerados idosos pacientes com 60 anos ou mais de idade, de acordo com a Política Nacional do Idoso ${ }^{7}$. Excluíram-se do estudo os idosos que se internaram em estado grave com forte ameaça à vida e aqueles com impossibilidade de comunicação.

A coleta de dados foi realizada entrevistando-se os idosos internados através da aplicação de formulários semiestruturados testados previamente (pré-teste realizado com cinco pacientes idosos hospitalizados), de forma individual à beira do leito no final do internamento (dia da alta ou véspera). Os itens do formulário foram aplicados oralmente, e à medida que os pacientes respondiam, os dados foram preenchidos por escrito pelos entrevistadores.

Os instrumentos de coleta de dados foram estruturados da seguinte forma:

(1) Formulário clínico-demográfico: questões destinadas à identificação dos participantes e ao registro de dados demográficos (procedência, sexo, idade, estado civil, cor da pele, escolaridade, situação ocupacional, número de filhos, número de pessoas residentes na casa, número de cômodos e renda familiar mensal);

(2) Formulário com questões relativas à satisfação do idoso, elaboradas pelos autores e pré-testado: perguntas sobre a capacidade do idoso em distinguir a equipe médica em relação à de enfermagem e outras categorias profissionais (sim/não); identificação dos membros da equipe de saúde - médico, enfermeiro, nutricionista, psicólogo, fisioterapeuta ( $\operatorname{sim} /$ não); satisfação quanto à atenção, educação e orientação da equipe médica e da de enfermagem; qualidade geral da equipe de enfermagem durante o dia e a noite; qualidade das refeições; conforto e limpeza do quarto (ruim/regular/bom/excelente); nível de incômodo por barulho na enfermaria (pouco incomodado/incomodado/muito incomodado); respeito à privacidade (sim/não); satisfação com os resultados do tratamento (ruim/regular/bom/excelente); tempo de hospitalização (menos de 1 semana/1-2 semanas/2-3 semanas/mais que 3 semanas); satisfação com o esclarecimento informacional sobre à doença que motivou a internação (sim/não); aquisição de informações sobre o tratamento medicamentoso recebido (sim/não).

$\mathrm{Na}$ estatística descritiva, foram determinadas as frequências relativas e absolutas referentes às variáveis qualitativas, assim como médias e desviospadrão das variáveis quantitativas. Na estatística inferencial foram usados os testes de qui-quadrado para as variáveis dicotômicas e de Mann-Whitney e de Kruskall-Wallis para as variáveis ordinais e intervalares. Para avaliar correlações entre variáveis ordinais e intervalares usou-se o teste de correlação linear de Spearman. A significância estatística adotada foi de $5 \%$. Empregou-se o programa estatístico SPSS 20.0 para Windows nas análises.

O projeto foi aprovado pelo Comitê de Ética em Pesquisa (CEP) do HULW (UFPB). Todos os pacientes que concordaram em participar da pesquisa assinaram o Termo de Consentimento Livre e Esclarecido aprovado pelo CEP sob CAAE n 310338914.4 .0000 .5183$. 


\section{RESULTADOS}

A idade dos 102 participantes variou de 60 a 90 anos, com média de 67,9 $( \pm 6,4)$ anos, $55,9 \%$ eram do sexo feminino, $46,1 \%$ eram casados ou mantinham união estável e $28,4 \%$ não eram alfabetizados (Tabela 1). Quanto ao estado civil, verificou-se que os solteiros, viúvos e separados/divorciados, formando o grupo sem conjugalidade, apresentaram frequência maior que os casados $(54 \%)$. A renda familiar média da amostra foi referida como de 1,85 $( \pm 0,8)$ salários-mínimos. A duração da internação está demonstrada na Tabela 2.

$\mathrm{Na}$ segunda parte do formulário de coleta de dados, buscou-se identificar a satisfação do idoso sobre a qualidade dos serviços recebidos durante sua internação. Primeiro, perguntou-se se os pacientes distinguiam quem era da equipe de médicos e quem era da equipe de enfermeiros, e cuja resposta foi dada afirmativamente por $86(84,3 \%)$ deles.

Tabela 1. Características sociodemográficas dos pacientes idosos internados nas enfermarias de clínica médica do Hospital Universitário Lauro Wanderley entre setembro de 2014 e março de $2015(n=102)$

\begin{tabular}{lrr}
\hline Variáveis & \multicolumn{3}{c}{ Frequências } \\
& \multicolumn{1}{c}{$\%$} \\
& \multicolumn{1}{c}{$\%$} \\
\hline Sexo & 45 & 44,1 \\
Masculino & 57 & 55,9 \\
Feminino & & \\
ldade & 70 & 68,6 \\
$60-69$ anos & 25 & 24,5 \\
70-79 anos & 7 & 6,9 \\
80 ou mais & & \\
Estado civil & 47 & 46,1 \\
Casados & 33 & 32,4 \\
Divorciados/Separados & 17 & 16,7 \\
Viúvos & 5 & 4,9 \\
Solteiros & & \\
Etnia & 42 & 41,1 \\
Brancos & 37 & 36,3 \\
Negros & 18 & 17,6 \\
Pardos & 5 & 4,9 \\
Outros & & \\
Escolaridade & 29 & 28,4 \\
$\quad$ Não alfabetizados & 58 & 56,9 \\
Fundamental & & \\
incompleto & 12 & 11,8 \\
$\quad$ Fundamental completo & 2 & 2,0 \\
$\quad$ Médio completo & 1 & 1,0 \\
$\quad$ Superior completo & & \\
Renda & 36 & 35,3 \\
1 Salário-mínimo & 50 & 49,0 \\
2 Salários-mínimos & 16 & 15,7 \\
3-4 Salários-mínimos & &
\end{tabular}


Situação Ocupacional

Aposentados

Autônomos

986,1

Assalariados

Tabela 2. Duração da internação dos pacientes idosos internados nas enfermarias de clínica médica do Hospital Universitário Lauro Wanderley entre setembro de 2014 e março de 2015 ( $n=102)$

\begin{tabular}{lcr}
\hline Duração da internação & \multicolumn{2}{c}{ Frequências } \\
& $\boldsymbol{f}$ & $\%$ \\
\hline Até 1 semana & 5 & 4,9 \\
1 a 2 semanas & 25 & 24,5 \\
2 a 3 semanas & 37 & 36,5 \\
Mais de 3 semanas & 35 & 34,3 \\
\hline
\end{tabular}

Quando questionados especificamente sobre a satisfação com a equipe médica, os pacientes responderam que esta foi "excelente" para $85,3 \%$ e boa para $14,7 \%$ dos casos. Não houve respostas "regular" e "ruim".

Quanto ao atendimento da equipe de enfermagem, que é composta por enfermeiros, técnicos e auxiliares de enfermagem, $80(78,4 \%)$ dos pacientes classificaram-no como "excelente" e $22(21,6 \%)$ como bom. Não houve diferença estatisticamente significativa na classificação da satisfação com a equipe de enfermagem entre os turnos diurno e noturno. As respostas quanto à satisfação com o atendimento médico correlacionaram-se de forma estatisticamente significativa com a satisfação com a equipe de enfermagem $(p=0,001)$, observando-se correlação positiva e de moderada magnitude (rho $=0,52)$.

Quando questionados se a equipe de saúde prestou esclarecimentos sobre a doença que motivou a internação, $82(80,4 \%)$ pacientes responderam que não obtiveram informações, enquanto que $92(90,2 \%)$ responderam que não receberam informações sobre o tratamento recebido. A obtenção ou não de esclarecimentos sobre a doença e ao tratamento também não se relacionou com a idade e com o grau de escolaridade dos pacientes entrevistados na nossa amostra. Houve diferença em relação ao sexo quanto à satisfação com as informações sobre a doença $(p=0,04)$, pois dos 20 pacientes que relataram que receberam informações, $75 \%$ eram mulheres.

Verificou-se que $92(90,2 \%)$ dos pacientes responderam que não obtiveram esclarecimentos por parte da equipe de saúde sobre os medicamentos que estavam recebendo durante a internação e, portanto, este foi um percentual ainda maior que o relacionado às respostas desfavoráveis quanto à obtenção de informações sobre a doença que motivou a internação.

Observou-se que $54,9 \%$ da nossa amostra consideraram bom o resultado do tratamento realizado durante a hospitalização. As respostas a este item do questionário e sobre a qualidade das refeições, conforto e limpeza das enfermarias, incômodo com ruídos e respeito à privacidade estão demonstradas na Tabela 3. 
Tabela 3. Respostas de pacientes idosos internados nas enfermarias de clínica médica do Hospital Universitário Lauro Wanderley entre setembro de 2014 e março de 2015 ( $n=102)$ sobre variáveis referentes ao atendimento hospitalar

\begin{tabular}{lcr}
\hline Variáveis & $\begin{array}{c}\text { Frequências } \\
\boldsymbol{f}\end{array}$ & $\%$ \\
\hline Qualidade das & & \\
refeições & 52 & 51,0 \\
$\quad$ Regular & 50 & 49,0 \\
$\quad$ Bom & & \\
Conforto e limpeza & & \\
dos quartos & 63 & 61,8 \\
$\quad$ Bom & 69 & 38,2 \\
$\quad$ Excelente & & \\
Incômodo com ruído & & \\
$\quad$ Pouco incomodado & 78 & 76,5 \\
$\quad$ Incomodado & 24 & 23,5 \\
Respeito à & & \\
privacidade & 102 & 100 \\
$\quad$ Sim & - & - \\
$\quad$ Não & & \\
Resultado do & & \\
tratamento & \\
$\quad$ Excelente & 42 & 41,2 \\
$\quad$ Bom & 56 & 54,9 \\
$\quad$ Regular & 4 & 3,9 \\
\hline
\end{tabular}

Observou-se correlação entre a satisfação com a enfermagem e a duração da permanência hospitalar $(p=0,01)$, porém foi uma associação positiva e de fraca magnitude ( $r h o=0,30)$, indicando que quanto maior a permanência, maior a satisfação.

\section{DISCUSSÃO}

A maior prevalência de pacientes do sexo feminino observada na amostra estudada é compatível com estudos sobre o perfil de internação de pessoas idosas em hospitais públicos no Brasil ${ }^{2,8}$. O maior percentual de mulheres na participação de pesquisas clínicas de amostragem não probabilística pode estar relacionado à maior longevidade feminina, ao maior autocuidado apresentado pelas mulheres e a sua maior busca ativa por atendimento em serviços de saúde 9 .

O percentual de pacientes viúvos, separados e solteiros foi superior ao observado por Siqueira et al. (2004), que verificaram frequência de $35,1 \%$ de idosos descasados entre os internados em um hospital universitário de São Paulo ${ }^{10}$. De acordo com Camarano e Ghaouri (2008), a viuvez e o aumento do número de divórcios contribuem para o crescimento das famílias unipessoais e, nesse caso, de idosos morando sozinhos -, situação que lhes confere maior 
risco, pela deterioração de suas condições de saúde ${ }^{11}$. Esses autores salientam que os idosos sem laços conjugais estão sob maior vulnerabilidade clínica e social. Contudo, 74 (72,5\%) dos entrevistados no nosso estudo tinham de um a quatro filhos, enquanto $97(95,1 \%)$ relataram ter de uma a sete pessoas convivendo com eles na mesma casa, e apenas $8(7,8 \%)$ moravam sozinhos.

A baixa escolaridade dos pacientes da nossa amostra é compatível com os resultados de estudos em que se analisou o perfil de indivíduos de todas as idades que procuraram os serviços de saúde pública no Brasil ${ }^{12,13}$. Pesquisa realizada em todo território brasileiro revelou que, para os usuários do Sistema Único de Saúde, houve predominância da baixa escolaridade e de nãoempregados $^{13}$. Considerando que $85,3 \%$ dos pacientes idosos entrevistados na nossa amostra do presente estudo não eram alfabetizados ou possuíam o ensino fundamental incompleto, e que o grau de escolaridade se reflete no senso crítico, a expectativa sobre a qualidade do atendimento hospitalar na amostra pode ser pequena. A baixa renda mensal dos pacientes e a sua baixa escolaridade, quando associadas, parecem estar agregadas ao perfil dos usuários do Sistema Único de Saúde, do qual são dependentes para que seus problemas de saúde sejam resolvidos ${ }^{9}$.

O resultado do presente estudo quanto à satisfação dos pacientes idosos com 0 atendimento hospitalar pela equipe médica foi positivo, observando-se elevada frequência de respondentes que referiram a atenção recebida como excelente. Este resultado foi superior ao encontrado por Cintra et al. (2012), em trabalho comparável ao nosso e realizado em um hospital universitário do interior do Mato Grosso, e em que 52,7\% dos usuários (média de idade de $55,7 \pm 18,1$ anos) consideraram-se "muito satisfeitos" enquanto $38,2 \%$ sentiramse "satisfeitos" com o atendimento médico ${ }^{14}$. Contudo, o nosso resultado foi semelhante ao observado em estudo realizado por Silva et al. (2004) em um hospital universitário de São Paulo, SP, observou-se que 96\% dos 126 pacientes entrevistados consideraram bom o atendimento médico recebido, dentre as alternativas eram bom, regular e ruim que lhes foram apresentadas ${ }^{15}$.

Na satisfação com a equipe médica, é imprescindível a adequada relação médico-paciente, cujo principal componente é a comunicação, em que o profissional esclarece o diagnóstico e o plano terapêutico ao paciente ${ }^{16}$, aspectos relacionados às perguntas feitas aos entrevistados do nosso estudo, especificamente em relação à atenção, educação e orientação percebidas sua doença e tratamento. Este resultado foi similar ao encontrado em estudo realizado com 476 pacientes hospitalizados entrevistados no momento da alta, em que se verificou que $83 \%$ ficaram satisfeitos com seu atendimento e $91 \%$ satisfeitos com a comunicação e com o tratamento médicos ${ }^{17}$.

A capacidade de os pacientes distinguirem a identificação funcional dos médicos e enfermeiros que os atendiam diferiu do que foi reportado em estudo desenvolvido em um hospital universitário do estado de São Paulo, em que a maioria dos 86 idosos internados em enfermarias clínicas e cirúrgicas reconheceu os médicos e a equipe de enfermagem como componentes da equipe de saúde que cuidava deles, porém 55,9\% não conseguiram informar a identidade de seu médico assistente ${ }^{18}$. Na nossa amostra, quando 
questionados sobre a auto-identificação da equipe, $79,4 \%$ dos idosos afirmaram que as pessoas que formavam a equipe revelaram sua identidade funcional para eles, como médicos, enfermeiros ou outro profissional do serviço.

A relação entre profissionais de saúde e pacientes tem sido considerada como o fator mais influente na satisfação do paciente com o atendimento ${ }^{19}$, cujo principal componente é a comunicação, na qual se inclui o esclarecimento sobre o diagnóstico e o plano terapêutico ao paciente por parte do profissional ${ }^{16,20}$. A satisfação do paciente é determinada principalmente pela atitude de respeito e empatia, mais ainda que de habilidades técnicas ${ }^{21}$. Boudreaux et al. (2000) observaram que 437 pacientes de um serviço de emergência consideraram que respeito, segurança e obtenção de instruções compreensíveis foram fatores associados com satisfação ${ }^{22}$. Sabe-se que uma má comunicação médico-doente/cuidador traduz-se em piores resultados em termos de satisfação e entendimento da informação recebida, resultando em cuidados de saúde inapropriados e pouco efetivos após a alta hospitalar ${ }^{23}$. Por outro lado, salienta-se nesse sentido que o nível de instrução também influencia a percepção sobre a comunicação em saúde ${ }^{24}$.

Quanto à satisfação com o atendimento prestado pela equipe de enfermagem, o resultado observado no presente estudo foi semelhante ao encontrado por Cintra et al. (2012) em um hospital universitário, onde 70,9\% dos pacientes idosos responderam que estavam "muito satisfeitos" com o atendimento da equipe de enfermagem, não havendo respostas desfavoráveis, de forma semelhante ao que foi observado no presente estudo ${ }^{14}$. Em outra pesquisa, entretanto, realizada em hospital na Etiópia, envolvendo 189 pacientes jovens (26,5 $\pm 15,1$ anos), contudo, a maioria declarou-se insatisfeita com o atendimento de enfermagem ${ }^{25}$, sugerindo que a idade, o país de origem ou a etnia podem ser fatores relacionados ao modo como os usuários dos hospitais relatam sua satisfação com o atendimento.

Respostas desfavoráveis foram observadas em relação à satisfação com as informações recebidas pela equipe de saúde sobre a doença que motivou o internamento e seu tratamento. Woldeyohanes et al. (2015) também observaram em uma amostra de pacientes hospitalizados que a maioria ficou insatisfeita com as informações recebidas sobre sua doença ${ }^{25}$. Não se observou diferença no nível de escolaridade entre os pacientes que afirmaram ter recebido e os que disseram não ter obtido esclarecimentos, como era de se esperar segundo o senso comum e considerando os resultados de estudos anteriores ${ }^{25,26}$.

Nas hospitalizações de idosos, segundo Veloso e Spindola (2005), falta preparo dos profissionais de saúde para exercer uma ação educativa, principalmente no momento da sua alta hospitalar ${ }^{27}$. Além disso, os usuários de hospitais públicos, de modo geral, não têm conhecimento de seus direitos ${ }^{28}$. Os pacientes de classes sociais menos favorecidas tendem a avaliar "positivamente" os serviços que lhes são prestados e a não entender o atendimento como um direito seu, considerando que o serviço recebido é um "favor" feito pela instituição à população ${ }^{29}$. Nesse sentido, Prochet (2005) observou em seu estudo de abordagem qualitativa que metade das respostas 
positivas atribuídas por $80,2 \%$ dos idosos hospitalizados ao atendimento que receberam, expressaram paralelamente algum tipo de sentimento negativo em relação à equipe de saúde ${ }^{18}$. Em outro estudo qualitativo em que se entrevistaram pacientes idosos internados em dois hospitais públicos no município de São Paulo, identificou-se que seus direitos não foram adequadamente atendidos, embora a maioria desses idosos, quando questionados sobre $\mathrm{o}$ atendimento recebido, contraditoriamente considerou-o como "ótimo" 30 .

O fator idade aparenta ser um aspecto importante nas respostas quanto à satisfação com o serviço de saúde, que parece ser maior em pacientes mais velhos $^{5}$, embora no nosso estudo a idade entre os idosos não tenha diferido em relação às respostas dos pacientes sobre sua satisfação com a equipe médica, de enfermagem e com as informações sobre medicamentos ou à doença que motivou a internação, resultado diverso do que foi encontrado em estudo semelhante ${ }^{31}$ realizado em outro país.

Em relação a diferenças entre os sexos, as mulheres do nosso estudo referiram maior satisfação com a informação recebida durante a hospitalização, o que é condizente com os resultados de estudo realizado com idosos internados em hospital dos Estados Unidos, em que as pacientes do sexo feminino relataram avaliações globais de satisfação substancialmente mais positivas do que os homens, embora para pacientes do sexo masculino mais velhos, a comunicação com médicos tenha sido mais influente em suas classificações de satisfação geral do que para pacientes idosas ${ }^{32}$. Em outros estudos, as mulheres também foram mais propensas a expressar melhor satisfação com o atendimento hospitalar do que os homens, mas em outros não foi encontrada relação entre sexo e satisfação ${ }^{25,33-35}$.

Em um estudo multicêntrico envolvendo 64.900 pacientes egressos de uma internação em enfermarias clínicas e cirúrgicas de vários hospitais dos Estados Unidos, com média de idade de 61 anos, $56 \%$ do sexo feminino, $84 \%$ brancos, observou-se que a satisfação com o atendimento recebido aumentou com a idade, sendo maior entre pacientes de 65 a 80 anos $^{26}$. No referido estudo, a satisfação também foi maior em pacientes com melhor saúde autorreferida. Também em outro estudo, os dois preditores mais fortes de satisfação para 533 pacientes egressos de uma hospitalização foram idade avançada e autopercepção de melhor estado de saúde ${ }^{31}$. Assim, os resultados dos estudos mencionados sugerem que a idade e a forma como os pacientes avaliam o próprio estado de saúde devem ser levados em conta na interpretação de dados sobre a satisfação dos pacientes hospitalizados.

Estudo transversal com 650 pacientes provenientes de enfermarias clínicas e cirúrgicas de quatro hospitais gerais da Espanha mostrou relação de diferentes características demográficas com aspectos específicos da avaliação do atendimento, como a correlação positiva entre nível de escolaridade e satisfação com conforto e limpeza, enquanto estado civil associou-se com nível de informação recebida ${ }^{36}$. A escolaridade e sexo conjuntamente também são referidos como um fator preditivo da satisfação, como reportado em outro estudo em que pacientes de menor escolaridade ficaram mais satisfeitos com o atendimento durante a hospitalização que pacientes de maior escolaridade ${ }^{37}$. 
Além dos fatores já confrontados há ainda outros aspectos envolvidos nesta relação entre satisfação do paciente e escolaridade. Há teorias desenvolvidas para explicar os determinantes psicossociais da satisfação do paciente ${ }^{4}$. $\mathrm{Na}$ "teoria do cumprimento", por exemplo, supõe-se que a satisfação depende da diferença percebida entre a expectativa do indivíduo e o que efetivamente ele recebe. Na "teoria maturacional", por outro lado, sugere-se que os indivíduos mais idosos tendem a se sentir mais satisfeitos com o cuidado recebido porque eles têm maior familiaridade, por experiência própria, com potenciais deficiências no sistema de prestação de cuidados de saúde ${ }^{26}$. Sendo uma variável multifacetada, a satisfação do paciente resulta de um julgamento que as pessoas fizeram refletindo sua experiência em circunstâncias específicas e nesse sentido, as experiências anteriores em serviços de saúde influenciam essa expectativa ${ }^{38}$.

Duração da permanência hospitalar é outro fator a ser considerado. Os intervalos de frequências observados em relação à duração da permanência hospitalar foram compatíveis com os de estudos anteriores sobre hospitalização de idosos no Brasil e no exterior ${ }^{2,39,40}$. No estudo de Hajifathali et al. (2008), pacientes cuja internação durou de 11 a 15 dias ficaram mais satisfeitos com o serviço prestado do que pacientes com uma internação de menos de cinco dias ${ }^{17}$. Nossos resultados nesse sentido, ao contrário do que se esperava, indicaram que quanto maior foi a permanência, maior foi a satisfação, mas esta foi classificada como excelente e boa na amostra, não havendo outras categorias de resposta (regular, ruim) para obtenção de inferência estatística. Mas, neste sentido, é possível que os pacientes que demoraram mais a receber alta possam ter se familiarizado mais com o atendimento hospitalar e tenham estabelecido uma melhor interação com a equipe de enfermagem.

Os resultados referentes com a alimentação oferecida durante a hospitalização divergem dos encontrados em outro estudo realizado no Brasil, em que 50,9\% dos respondentes mostram-se "satisfeitos" e $23,6 \%$ "muito satisfeitos" com as refeições do hospital ${ }^{10}$. Acredita-se, no entanto, que o fato de rotinas alimentares em hospitais, tanto quanto ao horário quanto ao tipo de comida, em geral, não são itens que promovam satisfação, pois são mais estritamente seguidas em função do quadro clínico e a idade dos pacientes. Contudo, de forma similar ao que foi encontrado no presente estudo, apenas um terço dos pacientes ficaram satisfeitos com a qualidade dos alimentos recebidos durante a internação hospitalar em outros dois estudos realizados fora do Brasil ${ }^{17,44}$. Quanto ao conforto e limpeza dos quartos das enfermarias, o resultado encontrado no nosso estudo foi semelhante ao observado por Cintra et al. (2012) no Brasil, em que 43,6\% dos usuários ficaram "satisfeitos" e 38,2\% "muito satisfeitos" quando questionados sobre a limpeza e higienização do quarto ${ }^{14}$.

Ressalta-se que as alternativas de resposta no questionário sobre se houve incômodo pelo ruído no ambiente foram "pouco incomodado", "incomodado" e "muito incomodado" e, portanto, nenhum paciente referiu que se sentia muito incomodado com ruídos nas enfermarias, que tinham quartos de dois leitos em um setor com 40 pacientes hospitalizados no período da realização da pesquisa. Pode-se inferir que, nesta amostra, a maioria dos pacientes idosos 
não percebeu o ruído ambiental como muito incômodo, mesmo que se considere que o nível de ruído de muitos dos hospitais brasileiros esteja acima dos padrões recomendados e possam acarretar respostas fisiológicas ao estresse, sobretudo em hospitais universitários, que contam com maior número de equipamentos e circulação de pessoas ${ }^{42,43}$.

Um achado inesperado foi o referente à percepção dos pacientes entrevistados de que sua privacidade foi respeitada, considerando as características do funcionamento de hospitais universitários, que possuem treinamento em serviço de alunos de graduação da área da saúde, médicos residentes e de especializações de outras áreas. Assim, ponderando que os pacientes entrevistados entenderam o conceito de privacidade nas enfermarias, depreende-se que esta pôde ser concretizada em um ambiente no qual geralmente faz parte da rotina a participação de estudantes da área da saúde e em que os pacientes são colocados, pelo menos, em parte, na condição temporária de "objeto de ensino" para os futuros profissionais da saúde ${ }^{44}$. Nessa condição, aos pacientes são impostos papéis caracterizados por acentuada dependência da equipe de saúde e espaço físico limitado ao seu quarto ou leito ${ }^{45}$.

Confrontando os resultados da presente pesquisa com dados publicados pela Ouvidoria do HULW sobre a satisfação da clientela internada em 2016 incluindo 368 pacientes adultos de todas as idades atendidos na instituição, observou-se semelhança nas respostas de itens sobre satisfação equivalentes: $88,6 \%$ dos respondentes consideraram bom ou ótimo o atendimento da equipe de saúde em relação à gentileza e tratamento de saúde recebido, enquanto $48,7 \%$ responderam que a higiene, a limpeza e a organização do hospital foram boas ou ótimas ${ }^{46}$. Esta última resposta apresentou frequência menor que a encontrada na nossa pesquisa, mas diferentemente da pergunta da nossa pesquisa, no levantamento feito pelo hospital esse item incluía também o termo "organização". Além disso, as amostras diferiram em relação à faixa etária.

É preciso considerar também que a satisfação do paciente não depende apenas da boa qualidade do serviço que lhes é prestado, e que a "insatisfação" pode se manifestar somente quando os desfechos clínicos dos doentes são negativos, ou seja, quando não houve melhora do seu quadro ou quando surgiram complicações. Na amostra estudada, contudo, não houve resposta "ruim" à pergunta sobre o resultado do tratamento hospitalar e apenas quatro pacientes entre os 102 entrevistados referiram que o resultado foi "regular".

A definição de qualidade no atendimento na área da saúde é considerada um aspecto de difícil análise, pela subjetividade inerente ao conceito, o que acaba por afetar a precisão exigida em termos de mensuração ${ }^{47}$. Salienta-se que a reflexão sobre qualidade implica pensar na prática dos serviços prestados ${ }^{48}$. Nesse contexto, a necessidade de construção de uma metodologia para avaliar a satisfação dos usuários do SUS advém essencialmente da carência de uma estratégia metodológica válida e confiável, que seja aceita por pesquisadores que trabalham com a avaliação da satisfação ${ }^{4}$. Em termos ideais, a maioria dos pacientes hospitalizados espera que haja limpeza nos quartos e enfermarias, uma equipe de saúde atenciosa, médicos competentes e comunicativos, 
participação própria em decisões sobre seu tratamento e um desfecho positivo com melhora dos seus sintomas e sinais clínicos ${ }^{38}$.

A concepção tradicional define um hospital universitário como uma instituição reconhecida oficialmente como uma clínica de ensino, estando submetido à supervisão das autoridades competentes da área da educação ${ }^{13,48}$. Nesse âmbito, como a alta do hospital é um ponto crítico na evolução de um paciente, pode levar a efeitos adversos e uma reinternação evitável, particularmente para pacientes idosos e que têm múltiplas comorbidades ${ }^{49}$. Por estas razões e pelo fato de os hospitais universitários serem centros de formação de recursos humanos e de desenvolvimento de tecnologia à área de saúde, a padronização dos elementos do processo de alta poderia minimizar lacunas em termos de qualidade e segurança que ocorrem quando os pacientes idosos voltam para suas casas, incluindo preenchimento de checklists focados em segurança sobre o uso de medicamentos, educação dos pacientes, assim como na melhor comunicação de médicos e enfermeiros com eles ${ }^{50}$.

As limitações do presente estudo são aquelas inerentes a um estudo de modelo transversal, com configuração específica e amostragem por conveniência, razões pelas quais seus resultados não podem ser generalizados para pacientes idosos de outros serviços hospitalares. Outro aspecto que representa uma limitação é o fato de que a satisfação dos pacientes foi abordada quantitativamente, mas com análise e interpretação das respostas usando-se categorias qualitativas (de ruim a excelente). Por isso, e em decorrência dos níveis elevados de satisfação identificados em diferentes estudos na área da saúde em hospitais públicos, existe a preocupação em se estabelecerem novas estratégias metodológicas para investigar em profundidade a opinião dos usuários ${ }^{15}$.

Inúmeros outros parâmetros psicossociais e clínicos relacionados não foram avaliados no presente estudo, não permitindo inferências quanto ao fato de a percepção sobre o atendimento recebido durante a internação estar ou não relacionada à gravidade clínica dos pacientes ou à sua saúde autorreferida, não sendo possível, portanto, avaliar a influência destas variáveis na satisfação com o atendimento. Por fim, neste estudo examinou-se a perspectiva apenas do paciente, não se avaliando a extensão em que as expectativas deste possam ter sido irrealisticamente baixas. Deve-se ressaltar também que as opiniões dos pacientes entrevistados não necessariamente refletem a situação do serviço de saúde, mas sim a sua percepção deles, o que implica a necessidade de reflexão sobre o significado dos resultados. Tais aspectos são um desafio a ser enfocado em pesquisas futuras.

\section{Referências}

1- Coelho Filho JM. (2000). Modelos de serviços hospitalares para casos agudos em idosos. Rev. Saúde Pública 2000; 34(6), 666-671 
2- Lima AP, Mantovani MF, Ulbrich EM, Zavadil ETS. Produção Científica sobre a Hospitalização de Idosos: Uma Pesquisa Bibliográfica. Cogitare Enferm 2009; 14(4),740-7

3- Cacique DB, Bacha AM, Grassioto OR, Oliveira HCO, Lima MT, FonsechiCarvasan GA. A avaliação da satisfação de pacientes internadas em um hospital universitário especializado na saúde materno-infantil: questionários e resultados globais. RAS 2002; 14 (57): 134-142

4- Esperidião MA, Trad LAB. Avaliação de satisfação de usuários: considerações teórico-conceituais. Cad. Saúde Pública 2006; 22 ( 6 ): 12671276

5- Imbelloni LE, Pombo IAN, Morais Filho GB. A diminuição do tempo de jejum melhora o conforto e satisfação com anestesia em pacientes idosos com fratura de quadril. Rev. Bras. Anestesiol 2015; 65 (2): 117-123.

6- Novaes MRCG, Lima ACV, Nogueira DY, Cerqueira F, Mello GF, Souza HS. Estudo etnográfico e de satisfação de profissionais e usuários do Programa de Saúde da Família em Samambaia, Distrito Federal. Comun Ciênc Saúde 2010; 21(4),289-300.

7- Brasil. Lei n. 8.842, de 04 de janeiro de 1994. Dispõe sobre a Política Nacional do Idoso, cria o Conselho Nacional do Idoso e dá outras providências [Internet]. Brasília; 1994. Disponível em: http://www.planalto.gov.br/ccivil 03/leis/l8842.htm. Acesso em: 09 ago. 2017.

8- Motta CCR, Hansel CG, Silva J. Perfil de internações de pessoas idosas em um hospital público. Rev. Eletr. Enf. 2010; 12(3):471-7

9- Pilger C; Menon MH, Mathias TAF. Características sociodemográficas e de saúde de idosos: contribuições para o serviço de saúde. Rev. Latino-Am. Enfermagem 2011; 19(5), 232-249.

10- Siqueira AB, Cordeiro RC, Perracini MR, Ramos LR. Impacto funcional da internação hospitalar de pacientes idosos. Rev. Saúde Pública 2004; 38(5): 687-69.

11- Camarano A, Ghaouri SK. Famílias com idosos: ninhos vazios. Anais do $13^{\circ}$ Encontro da Associação Brasileira dos Estudos Populacionais. Ouro PretoMG - Brasil. 2008. Disponível em:

http://www.abep.nepo.unicamp.br/docs/anais/pdf/2002/Com ENV ST23 camar ano texto.pdf. Acesso em: 10 ago. 2017

12- Ribeiro MCSA. Perfil sociodemográfico e padrão de utilização de serviços de saúde para usuários e não usuários do SUS - Pnad 2003. Ciênc Saúde Coletiva 2006; 11(4): 1011-22

13- Gouveia GC, Souza WV, Luna CF, Souza-Júnior PRB, Szwarcwald CL. Satisfação dos usuários do sistema de saúde brasileiro: fatores associados e diferenças regionais. Rev. bras. epidemiol. 2009; 12 (3): 281-296. 
14- Cintra RF, Gonçalves LP, Dias B, Vieira SFA, Pacagnan MN, Dalmas JC. A Satisfação dos Usuários sobre os Serviços do Sistema de Saúde Pública Brasileiro: um Estudo no Hospital Universitário Federal. V Encontro da Divisão de Administração Pública da ANPAD - V EnAPG, 2012

15- Silva LMP, Muccioli C, Belfort JR. Perfil socioeconômico e satisfação dos pacientes atendidos no mutirão de catarata do Instituto da Visão - UNIFESP. Arq. Bras. Oftalmol 2004; 67(5): 737-744

16- Sucupira AC. A importância do ensino da relação médico-paciente e das habilidades de comunicação na formação do profissional de saúde. Interface (Botucatu) 2007; 11 (23), 624-627

17- Hajifathali A, Ainy E, Jafari H, Moghadam MN, Kohyar E, Hajikaram S. Inpatient satisfaction and its related factors in Taleghani University Hospital, Tehran, Iran. Pak J Med Sci(2008); 24 (2), 274-277

18- Prochet TC. A busca da assistência humanizada: a percepção do idoso hospitalizado. Interface - Comunic, Saúde, Educ 2005; 9 (16): 185-90

36- Quintana JM, González N, Bilbao A, Aizpuru F, Escobar A, Esteban C. Predictors of patient satisfaction with hospital health care. BMC Health Serv Res. 2006; 16 (6):102-106.

19- Hall JA, Dornan MC. Patient socio-demographic characteristics as predictors of satisfaction with medical care: a meta-analysis. Soc Sci Med 1990; 30(1), 811-818

20- Lee K, Kasper JD. Assessment of medical care by elderly people: general satisfaction and physician quality. Health Serv Res 1998; 32 (1), 741-758.

21- Cheng SH, Yang MC \& Chiang TL. Patient satisfaction with and recommendation of a hospital: effects of interpersonal and technical aspects of hospital care. Int J Qual Health Care. 2003; 15 (4), 345-55

22- Boudreaux ED, Ary RD, Mandry CV, McCabe B. Determinants of patient satisfaction in a large, municipal ED: the role of demographic variables, visit characteristics, and patient perceptions. Am J Emerg Med 2000; 18(1). 394-400

23- Lobão WM, Menezes IG. Construction and content validation of the scale of predisposition to the occurrence of adverse events. Rev. Latino-Am.

Enfermagem 2012; 20(4),796-803

24- Badziak RPF, Moura VEV. Determinantes Sociais da Saúde: Um Conceito para Efetivação do Direito à Saúde. R. Saúde Públ. Santa Cat, 2010; 3(1), 6979.

25- Woldeyohanes TR, Woldehaimanot TE, Kerie MW, Mengistie MA, Yesuf EA. Perceived patient satisfaction with in-patient services at Jimma University Specialized Hospital, Southwest Ethiopia. BMC Res Notes 2015; 8 (1):285. 
26- Jaipaul CK, Rosenthal GE. Are older patients more satisfied with hospital care than younger patients? J Gen Intern Med. 2003; 18 (1), 23-30.

27- Veloso RC \& Spindola T. A percepção do cliente hospitalizado acerca de seus direitos e deveres. Rev Enferm Uerj 2005; 13 (1);38-43

28- Meirelles, BHS, Arruda, C, Simon, E, Vieira, FMA, Cortezi, MDV. \& Natividade, M. S. L. Condições associadas à qualidade de vida de idosos com doenças crônicas. Cogitare Enferm 2010; 15 (3),433-440

29- Coloço RA, Holanda LD, Portero-Mclellan KC. Determinantes do grau de satisfação de pacientes internados referente a refeições oferecidas em um hospital universitário. Rev. Ciênc. Méd. 2009; 18 (3), 121-130

30- Ferreira AMT, Derntl AM. Ouvindo o idoso hospitalizado: direitos envolvidos na assistência cotidiana de enfermagem. In: Pessini, L, Barchifontaine CP. (Org.). Bioética e longevidade humana, 2006

31 - Nguyen Thi PL, Briançon S, Empereur F \& Guillemin F. Factors determining inpatient satisfaction with care. Soc Sci Med 2002; 54(4):493-504

32- Chumbler NR, Otani K, Desai SP, Herrmann PA, Kurz RS. Hospitalized Older Adults' Patient Satisfaction. Inpatient Care Experiences 2016; 6 (2): 1-7

33- Alasad JA, Ahmad MA. Patients' satisfaction with nursing care in Jordan. Int J Health Care Qual Assur 2003; 16 (1), 279-285.

34- Crow R, Gage H, Hampson S, Hart J, Kimber A, Storey L et al. The measurement of satisfaction with healthcare: implications for practice from a systematic review of the literature. Health Technol Assess 2002; 6(32),1-244

35- Flynn S. Nursing effectiveness: an evaluation of patient satisfaction with a nurse led orthopaedic joint replacement review clinic. J Orthop Nurs. 2005; 9:156-165

36- Quintana JM, González N, Bilbao A, Aizpuru F, Escobar A, Esteban C. Predictors of patient satisfaction with hospital health care. BMC Health Serv Res 2006; 16 (6):102-106

37- Al-Doghaither $\mathrm{AH}$. Inpatient satisfaction with physician services at King Khalid University Hospital, Riyadh, Saudi Arabia. East Mediterr Health J 2004; 10(3): 358-64

38- Bowling, A. Evaluating health services: multidisciplinary collaboration. In: Research methods in health: investigating health and health services.

Buckingham, Philadelphia: Open University Press, 2012, pp. 5-16.

39- Amaral ACS, Coeli CM, Costa MCE, Cardoso VS, Toledo ALA, Fernandes CR. Perfil de morbidade e de mortalidade de pacientes idosos hospitalizados. Cad Saúde Pública 2004; 20(6): 1617-26 
40- Guerrero L, Gascón CA. Variáveis biopsicossociais relacionadas à duração de hospitalização em idosos. Rev. Latino-Am. Enfermagem 2011; 19 (6):13771384

41- Annemieke PB, Andrew GHT. Predicting and comparing patient satisfaction in four different model of health care across a nation. Social Sciences \& Medicine 2006; 63(1): 1671-83

42- Filus WA, Pivatto LF, Fontoura FP, Koga MRV, Albizu EJ, Soares VMN. Ruído e seus impactos nos hospitais brasileiros: uma revisão de literatura. Rev. CEFAC 2014; 16 (1): 307-317

43- Pereira RP, Toledo RN, Amaral JLG, Guilherme A. Qualificação e quantificação da exposição sonora ambiental em uma unidade de terapia intensiva geral. Rev Bras Otorrinolaringol 2003; 69(6): 766-71.

44- Gonçalves ER, Verdi MIM. Os problemas éticos no atendimento a pacientes na clínica odontológica de ensino. Ciênc. saúde coletiva 2007; 2 (3):755-764

45- Oliveira AML, Guirardello EB. Satisfação do paciente com os cuidados de enfermagem: comparação entre dois hospitais. Rev. esc. enferm. USP 2003; 40 (1): $71-77$

46- Universidade Federal da Paraíba. Hospital Universitário Lauro Wanderley/Ebserh. Pesquisas de Satisfação. Disponível em: http://www.ebserh.gov.br/documents/220250/628973/RESULTADO_PESQUIS A_OUVIDORIA.pdf/ffca3d46-315b-4459-bbf7-9794bf07b05a. Acesso em: 08 set. 2017.

47- Löbler ML. A utilização da ciência cognitiva nos trabalhos científicos da área de sistemas de informações e apoio à decisão: revelações do estado da arte. READ-UFRGS 2003; 9 (31),1-20

48- Mezomo JC. Qualidade na relação hospital-paciente. Revista Hospital.Adm. e Saúde 1993; 17(1): 17

49- Medici AC. Hospitais universitários: passado, presente e futuro. Rev. Assoc. Med. Bras. 2001; 47( 2 ):149-156

50- Halasyamani L, Kripalani S, Coleman E, Schnipper J, van Walraven C, Nagamine J. Transition of care for hospitalized elderly patients--development of a discharge checklist for hospitalists. J Hosp Med 2006; 1 (6): 354-60

Recebido: 10 agosto 2017. Publicado: 18 setembro 2017

Correspondência: Profa. Rilva Lopes de Sousa-Muñoz. Centro de Ciências Médicas / Universidade Federal da Paraíba. Campus I, Jardim Universitário, 
s/n, Castelo Branco - João Pessoa-PB. CEP: 58050-000. E-mail:

rilva@ccm.ufpb.br

Conflito de Interesses: os autores declararam não haver conflito de interesses

(C) This is an Open Access article distributed under the terms of the Creative Commons Attribution License, which permits unrestricted use, distribution, and reproduction in any medium, provided the original work is properly cited 